\section{To tPA or Not to tPA, That Is the Question}

(D)T.M. Leslie-Mazwi, (DR.V. Chandra, and (D).A. Hirsch

$F^{\circ}$ or decades, the acute treatment of stroke has centered on delivery of intravenous recombinant tissue plasminogen activator. Endovascular stroke therapy was historically dogged by a dearth of high-level evidence supporting its application. Refinements in imaging selection, improved treatment logistics, and increased reperfusion rates came together in 2015 with the publication of multiple randomized trials that changed that, unequivocally identifying the benefit of endovascular therapy combined with medical management over medical management alone for patients with proximal large vessel occlusions (LVO). ${ }^{1-5} \mathrm{With}$ a number needed to treat of merely 2.6 to reduce disability (measured by the modified Rankin score), endovascular stroke therapy is now arguably one of the most effective treatments in medicine. ${ }^{6}$

Attention therefore turns to refinements and the remaining gaps in knowledge for this powerful therapy. This requires examination of every aspect of the care of patients with LVO. It is in this context that the role of IV tPA in patients with LVO undergoing endovascular therapy has come under scrutiny. Specifically, should IV tPA be administered at all?

\section{The State of the Science}

In the original 5 published randomized controlled trials (RCTs) demonstrating the superiority of endovascular therapy over medical management alone, IV tPA had no impact on the endovascular therapy results. ${ }^{1-5}$ Both the Multicenter Randomized Clinical trial of Endovascular treatment for Acute ischemic stroke in the Netherlands (MR CLEAN) ${ }^{1}$ and Endovascular Revascularization With Solitaire Device Versus Best Medical Therapy in Anterior Circulation Stroke Within 8 Hours (REVASCAT) ${ }^{4}$ required IV tPA failure before randomization for endovascular treatment, selecting patients likely to do poorly with isolated medical management. However, the remaining 3 trials (Endovascular Treatment for Small Core and Proximal Occlusion Ischemic Stroke [ESCAPE], Extending the Time for Thrombolysis in Emergency Neurological Deficits-Intra-Arterial [EXTEND-IA], and Solitaire With the Intention For Thrombectomy as PRIMary Endovascular Treatment for Acute Ischemic Stroke [SWIFT PRIME] $)^{2,3,5}$ were more selective and had no such requirement; 2 of these (EXTEND-IA and SWIFT PRIME) administered IV tPA to all participants. More recently the Trial and Cost Effectiveness Evaluation of Intra-Arterial Thrombectomy in Acute Ischemic Stroke [THRACE] trial, with wide inclusions, also reported similar findings: outcome was independent of IV tPA status. ${ }^{7}$

The HERMES meta-analysis aggregates these collective data at the individual patient level (excluding THRACE). ${ }^{6}$ For the endovascular cohort, 526/634 (83\%) received tPA, compared

http://dx.doi.org/10.3174/ajnr.A5263 with $87 \%$ of the control population. From the thrombectomy group, 108 patients were ineligible for IV tPA of 188 total. There was no treatment-effect heterogeneity across a range of prespecified variables; the administration of tPA caused neither benefit nor harm. The authors concluded that endovascular therapy should be pursued irrespective of tPA eligibility or status.

These are specific trial populations, and even in aggregate, there are reasons that generalizing to patients in everyday practice requires caution. However, several recent publications have explored this in typical clinical scenarios.

Weber et $\mathrm{al}^{8}$ retrospectively analyzed patients treated with combination therapy with IV tPA and thrombectomy $(n=105)$ compared to patients receiving thrombectomy alone $(n=145)$ during a 14-month period at a tertiary neurovascular center. Administration of IV tPA added a 36-minute delay in picture-topuncture times. This finding is striking in an efficient, high-volume center. No significant differences existed in 90-day outcomes (modified Rankin Scale score, $0-2 ; 35.2 \%$ versus $40 \%$; $P=.444$ ). Similarly, TICI 2b/3 (73.8\% versus $73.1, P=.952)$ and symptomatic hemorrhage rates $(5.9 \%$ versus $3.5 \%, P=.387)$ were equivalent.

A similar experience is reported during nearly 6 years in a high-volume stroke center. ${ }^{9}$ Like the groups in Weber et al, ${ }^{8}$ treatment decisions were individualized by the multidisciplinary treating team, excepting clear exclusions to IV tPA use. They reviewed 239 patients with anterior circulation stroke treated with mechanical thrombectomy alone and identified 40 IV tPA-eligible patients who were treated with thrombectomy alone. Multivariate matched-pairs analysis was used to compare these patients with controls treated with combination therapy. There were no significant differences in 90-day favorable outcome (mRS, $0-2 ; 42.5 \%$ versus $42.5 \%$; $P=1.0$ ), successful reperfusion (TICI $2 \mathrm{~b} / 3,87.5 \%$ versus $77.5 ; P=.39$ ), or symptomatic intracerebral hemorrhage ( $2.5 \%$ versus $2.5 \% P=1.0$ ). The patients who went directly to thrombectomy had lower rates of asymptomatic intracranial hemorrhage ( $12.5 \%$ versus $35 \%, P=.023$ ) compared with those receiving bridging IV tPA.

Most recently, Coutinho et $\mathrm{al}^{10}$ published an analysis of 2 large prospective clinical trials, Solitaire FR Thrombectomy for Acute Revascularization (STAR) and Solitaire With the Intention for Thrombectomy (SWIFT), further questioning the role of IV tPA for patients with LVO eligible for thrombectomy. ${ }^{10}$ The authors analyzed 291 patients, 55\% treated with combination therapy compared with thrombectomy alone. The groups were well-matched except that the baseline Alberta Stroke Program Early CT Score favored the combination therapy group ( 8 versus $9, P=.4$ ). Outcomes between the 2 groups were the same at 90 days, as was symptomatic hemorrhage. Technical considerations were identical between groups, with no difference in clot fragmentation (measured by emboli to new territory). The authors concluded that IV tPA and thrombectomy offered no obvious benefit over thrombectomy alone. ${ }^{10}$ 


\section{IV tPA in Patients with LVO}

We have previously published on this topic, outlining the arguments both for and against IV tPA in this setting. ${ }^{11}$ IV tPA has distinct positives: It may produce recanalization without the need for mechanical thrombectomy. It may enhance the success of thrombectomy, by both making clot extraction easier and lysing smaller fragments that may embolize distally during the procedure. It may prevent microvascular thrombosis, maintaining cerebral perfusion distal to the occlusion. Finally, it offers a chance (albeit small) at recanalization for those patients who, for technical or logistic reasons, are unable to undergo mechanical thrombectomy or in whom this is delayed. ${ }^{11}$

The arguments against the use of tPA are similarly varied. IV tPA has limited efficacy in the presence of large-volume clot, with an efficacy range of $8 \%-32 \%$, depending on the occlusion level. Administration of tPA produces delays, both in the act of delivering the drug and $\mathrm{PAA}$ prioritization in the care delivery system. In comprehensive centers, thrombectomy may be accomplished before the tPA infusion has been completed. Moreover, the potential for hemorrhage in patients who receive IV tPA is difficult to ignore, despite the equivalent symptomatic hemorrhage rates in recent endovascular trials. ${ }^{11}$ At this stage, there is no clear clinical benefit or harm incurred by administering IV tPA. However, the routine use of IV tPA for patients with LVO does erode the overall health care value.

Both resource-intensive and expensive, endovascular stroke treatment makes an attractive target for organizations aiming to control expenditure. Maximizing value through cost-effectiveness and cost-utility, therefore, becomes a priority. ${ }^{12}$ One prominent question that remains to be answered in this new landscape of endovascular stroke therapy is about the most cost-effective method to deliver this care within our evermore-constrained health care budget. Value is best viewed as the relationship between the cost of the treatment and outcome. In patients with LVO, this references overall expenditure (from onset to return to the community) weighted against the eventual patient outcome.

A recent cost-utility analysis using data with contemporary cost information found that combination therapy is approximately $\$ 17,000$ more expensive than IV tPA alone in initial hospital costs. ${ }^{13}$ By 90 days, this difference had shrunk to $\$ 12,000$ because of an average of $\$ 5000$ saved in the combination therapy cohort through earlier hospital discharge and reduced rehabilitation costs. When lifetime projections were incorporated, however, mechanical thrombectomy was economically dominant, with substantial overall cost savings of more than $\$ 23,000$ and both life-expectancy and quality-adjusted life expectancy gains. ${ }^{13}$ Effort to reduce the initial cost of thrombectomy treatment will further cement this economic dominance. The direct cost of IV tPA in the United States approximates $\$ 7000 / 100-\mathrm{mg}$ vial. This reflects only the actual price of the drug and not the additional ancillary expenses of delivering it. This expense represents an area of potential savings for patients treated with combination therapy. Indeed, the questions may well be more nuanced than "Does tPA provide any value?" It may be more appropriate to ask, "In select patients, does IV tPA provide $\$ 7000$ worth of value?”
A similar outcome at reduced cost improves value. Therefore, the concern that IV tPA adds little benefit to the patient with LVO should be evaluated in a randomized fashion. The RCT design would be pragmatic: direct to thrombectomy versus combination therapy in patients presenting early to centers with neuroendovascular capability. This would have important implications for future stroke treatment as episodic care, alternative payment, and physician-focused payment models grow increasingly important. Several trials have been proposed, and while published protocols are lacking, these trials are close to commencing.

The implications of a randomized trial proving that the addition of IV tPA carries no additional benefit for selected patients will be momentous. Resistance may be anticipated, but a welldesigned trial would win over many in the stroke neurology community, overcoming the ethics of withholding a proved treatment. A particular concern will be to prevent undue interruption of current tPA delivery networks, which must be protected for the general stroke population. A randomized trial will raise these and more questions. The stroke community should embrace the opportunity.

Disclosures: Joshua A. Hirsch—UNRELATED: Consultancy: Medtronic, Codman Neurovascular, CareFusion, Globus, Comments: Medtronic, ongoing spine; Codman Neurovascular, ongoing Data and Safety Monitoring Board; CareFusion, taught spine-related procedures in a single educational event; Globus, single consulting event.

\section{REFERENCES}

1. Berkhemer OA, Fransen PS, Beumer D, et al. A randomized trial of intraarterial treatment for acute ischemic stroke. NEngl JMed 2015; 372:11-20 CrossRef Medline

2. Campbell BC, Mitchell PJ, Kleinig TJ, et al; EXTEND-IA Investigators. Endovascular therapy for ischemic stroke with perfusion-imaging selection. N Engl J Med 2015;372:1009-18 CrossRef Medline

3. Goyal M, Demchuk AM, Menon BK, et al; ESCAPE Trial Investigators. Randomized assessment of rapid endovascular treatment of ischemic stroke. N Engl J Med 2015;372:1019-30 CrossRef Medline

4. Jovin TG, Chamorro A, Cobo E, et al; REVASCAT Trial Investigators. Thrombectomy within 8 hours after symptom onset in ischemic stroke. N Engl J Med 2015;372:2296-306 CrossRef Medline

5. Saver JL, Goyal M, Bonafe A, et al; SWIFT PRIME Investigators. Stent-retriever thrombectomy after intravenous t-PA vs. t-PA alone in stroke. N Engl J Med 2015;372:2285-95 CrossRef Medline

6. Goyal M, Menon BK, van Zwam WH, et al; HERMES collaborators. Endovascular thrombectomy after large-vessel ischaemic stroke: a meta-analysis of individual patient data from five randomised trials. Lancet 2016;387:1723-31 CrossRef Medline

7. Bracard S, Ducrocq X, Mas JL, et al; THRACE investigators. Mechanical thrombectomy after intravenous alteplase versus alteplase alone after stroke (THRACE): a randomised controlled trial. Lancet Neurol 2016;15:1138-47 CrossRef Medline

8. Weber R, Nordmeyer H, Hadisurya J, et al. Comparison of outcome and interventional complication rate in patients with acute stroke treated with mechanical thrombectomy with and without bridging thrombolysis. J Neurointerv Surg 2017;9: 229-33 CrossRef Medline

9. Broeg-Morvay A, Mordasini P, Bernasconi C, et al. Direct mechanical intervention versus combined intravenous and mechanical intervention in large artery anterior circulation stroke: a matchedpairs analysis. Stroke 2016;47:1037-44 CrossRef Medline

10. Coutinho JM, Liebeskind DS, Slater LA, et al. Combined intravenous thrombolysis and thrombectomy vs thrombectomy alone for acute 
ischemic stroke: a pooled analysis of the SWIFT and STAR studies. JAMA Neurol 2017;74:268-74 CrossRef Medline

11. Chandra RV, Leslie-Mazwi TM, Mehta BP, et al. Does the use of IV tPA in the current era of rapid and predictable recanalization by mechanical embolectomy represent good value? J Neurointerv Surg 2016;8:443-46 CrossRef Medline

12. Hirsch JA, Harvey HB, Barr RM, et al. Sustainable growth rate repealed, MACRA revealed: historical context and analysis of recent changes in Medicare physician payment methodologies. AJNR Am J Neuroradiol 2016;37:210-14 CrossRef Medline

13. Shireman TI, Wang K, Saver JL, et al; SWIFT-PRIME Investigators. Cost-effectiveness of Solitaire stent retriever thrombectomy for acute ischemic stroke: results from the SWIFT-PRIME trial (Solitaire With the Intention for Thrombectomy as Primary Endovascular Treatment for Acute Ischemic Stroke). Stroke 2017;48:379-87 CrossRef Medline 